\title{
Vortices in a rotating Bose-Einstein condensate under extreme elongation in a harmonic plus quartic trap
}

\author{
C. C. Huang, C. H. Liu, and W. C. Wu \\ Department of Physics, National Taiwan Normal University, Taipei 11650, Taiwan
}

\begin{abstract}
The behaviors of a rapid rotating Bose-Einstein condensate under extreme elongation in a 2D anisotropic harmonic plus quartic trap are investigated. Due to the quartic trap, the system remains stable at high rotating velocity, $\Omega \geq \omega_{\perp}\left(\omega_{\perp}\right.$ is the radial harmonic trap frequency), and vortex lattices form even in the absence of the repulsive $s$-wave interaction $(g)$. When $g$ is present, the interplay between $g$ and the quartic trap potential can lead to rich vortex lattice transition states as a function of $\Omega$, to which vortex lattices vanish eventually at some higher $\Omega$.

PACS numbers: 03.75.Hh, 32.80.Pj, 03.65.-w
\end{abstract}

\section{INTRODUCTION}

In recent years vortices in a rotating Bose-Einstein condensate (BEC) have been extensively studied theoretically and experimentally. Rotating condensates are typically confined in a harmonic potential such that large vortex arrays are obtained when angular velocity $\Omega$ is smaller than but approaching to the radial trap oscillator frequency $\omega_{\perp}$. For instances in Refs. [1 7], a single vortex state or a state of several hundreds of vortices have been successfully created. Theoretically, the behaviors of the rapid rotating BEC systems are usually studied based on the lowest Landau level (LLL) approximation [8 [13]. When angular velocity is greater than the radial trap oscillator frequency, $\Omega>\omega_{\perp}$, the system becomes unstable due to the ineffectiveness of confinement.

In the experiment of Bretin et. al [14], a quartic trapping potential has been successfully created. Several groups then consider the type of trap potential $V(x, y)=\frac{1}{2} m \omega_{\perp}^{2} r_{\perp}^{2}+\frac{1}{4} u r_{\perp}^{4}$, where $m$ is the atom mass, $u$ is the strength of the quartic potential, and $r_{\perp}^{2}=x^{2}+y^{2}$ 15 19]. With this quartic potential, due to the effect of strong confinement, one thus can study the rotating Bose condensates at higher angular velocities, $\Omega \geq \omega_{\perp}$. It is understood that the centrifugal force, varying as $\Omega^{2} r_{\perp}$, can always be compensated by the trapping force varying as $-\left(m \omega_{\perp}^{2} r_{\perp}+u r_{\perp}^{3}\right)$.

Rotating BECs have also been studied under an anisotropic harmonic trap for a quasi-two-dimensional (2D) system 20, 21]. Moreover, by manipulating the anisotropic trap frequencies associated with the rotating velocity, quasi-2D rotating $\mathrm{BEC}$ under the anisotropic harmonic trap can be reduced to the limit of extreme elongation 22 24]. In Ref. 22] the authors have studied the roton-maxon excitation spectrum in a rapid rotating weakly interacting BEC under extreme elongation in a $2 \mathrm{D}$ anisotropic harmonic trap. When the interaction or the angular velocity increases, the system could undergo a second-order quantum transition to a state of a periodic structure - rows of vortices [22]. Similar transitions have also been investigated in Refs. 23, 24]. A common finding is that the larger the interaction or angular velocity is, the more the vortex lattice row number is.

Inspired by the works mentioned above [14, 22 24], it is of particular interest to study how the rapid rotating $\mathrm{BEC}$ behaves under the extreme elongation if a quartic trapping potential is added. With this goal, this paper considers the following effective $2 \mathrm{D}$ trap potential

$$
V(x, y)=\frac{1}{2} m\left[\omega_{x}^{2} x^{2}+\omega_{y}^{2} y^{2}\right]+\frac{1}{4} u x^{4},
$$

where, with the strength $u$, the anharmonic quartic potential acts on the $x$-axis only. By adding the quartic potential along the $x$ axis only, the effect of the anharmonic trap for the extreme elongated system can be seen in a more transparent way (effective 1D problem). In fact, the kind of effective trap (11) can be realized in a realistic experiment. One can consider a quasi-2D system that is confined in the $x y$ plane by a harmonic trap of relatively large $\omega_{z}$. Now, consider that an extra isotropic 2D quartic trap, $V_{4} \sim\left(x^{2}+z^{2}\right)^{2}$, is added and suppose that the rotating angular frequency is purely along the $z$ direction. Since the system is already confined in the $x y$ plane, any quartic trap effect in the $z$ direction will simply enhance the confinement of the system in the $x y$ plane. This means effectively, $V_{4} \sim x^{4}$.

With the quartic trapping potential (10) which is introduced to stabilize the system against decay at higher rotation velocities $(\Omega)$, one can then study the system even for $\Omega \geq \omega_{x}$. It will be shown that in the limit of no interaction, ground state forms no vortex lattice at smaller $\Omega$ and when $\Omega$ becomes larger, one row of vortex lattice will form. Vortex lattice will melt eventually at some larger $\Omega$. When the interaction is turned on, melting of the vortex lattice at some higher $\Omega$ is also found. Nevertheless, rich transition states occur for the vortex lattices. The transition states, arising due to the instability at a critical interaction strength or a critical rotation velocity, were first studied in Ref. 22] in an elongated system without the quartic potential. One will see that a similar instability leading to the formation of a periodic structure of vortex rows occurs in the current context. Of most interest, a parameter labeled by $N_{c}$ which corresponds to number of terms involved in the $k$-sum of the wavefunction is intimately related to the number of 
vortex rows [22].

The paper is organized as follows. In Sec. II] we outline the GP energy functional essential for a rapid rotating BEC system under extreme elongation in a 2D anisotropic harmonic plus quartic trap. Details about how the ground-state wave function is obtained and how the total energy is minimized are given. In Sec. III we study the properties of the system without the interaction. This section is devoted to fully understand the effect of the quartic potential. It is shown that the behaviors of the system with a quartic potential are quite different compared to those of no quartic potential. In Sec. IV. effect of the $s$-wave interaction $g$ is studied, which is treated by the perturbation method. It will be shown that the interplay between $g$ and the quartic trap potential can lead to rich vortex lattice transition states as a function of $\Omega$, to which vortex lattices vanish eventually at some higher $\Omega$. Sec. $\mathrm{V}$ is a brief conclusion.

\section{GP ENERGY FUNCTIONAL}

The GP energy functional of a rapid rotating, quasi-2D BEC system can be given by

$E=\int d x d y \Psi_{0}^{*}\left[-\frac{\hbar^{2} \nabla^{2}}{2 m}+V(x, y)+\frac{g_{2 \mathrm{D}}}{2}\left|\Psi_{0}\right|^{2}-\boldsymbol{\Omega} \cdot \mathbf{L}\right] \Psi_{0}$,

where the trap potential $V(x, y)$ was given in Eq. (11), $\Psi_{0}=\Psi_{0}(x, y)$ is the wave function, $g_{2 \mathrm{D}} \equiv$ $4 \pi \hbar^{2} a_{s} /\left(m a_{z} \sqrt{2 \pi}\right)$ is the effective $2 \mathrm{D} s$-wave interaction with $a_{s}$ the $s$-wave scattering length and $a_{z} \equiv \sqrt{\hbar / m \omega_{z}}$ associated with the harmonic trap frequency $\omega_{z}$ [25], $\boldsymbol{\Omega}$ is the rotation angular velocity, and $\mathbf{L}$ is the angular momentum. When $\Omega=\Omega \hat{z}$, the rotating term becomes

$$
-\Omega \Psi_{0}^{*} L_{z} \Psi_{0}=i \hbar \Omega \Psi_{0}^{*}\left[x \frac{\partial}{\partial y}-y \frac{\partial}{\partial x}\right] \Psi_{0} .
$$

Eq. (2) can also be written as

$$
\begin{aligned}
E & =\int d x d y \Psi_{0}^{*} \\
& \times\left[\frac{\left(-i \hbar \nabla+m \mathbf{r}_{\perp} \times \Omega\right)^{2}}{2 m}+V_{\mathrm{eff}}(x, y)+\frac{g_{2 \mathrm{D}}}{2}\left|\Psi_{0}\right|^{2}\right] \Psi_{0},
\end{aligned}
$$

where $\mathbf{r}_{\perp}=(x, y)$ and $V_{\text {eff }}(x, y) \equiv(m / 2)\left[\left(\omega_{x}^{2}-\Omega^{2}\right) x^{2}+\right.$ $\left.\left(\omega_{y}^{2}-\Omega^{2}\right) y^{2}\right]+(u / 4) x^{4}$. For the part of the anisotropic harmonic trap, trap frequencies can be written as $\omega_{x}^{2} \equiv$ $\omega_{0}^{2}+\Lambda^{2}$ and $\omega_{y}^{2} \equiv \omega_{0}^{2}-\Lambda^{2}$. If one sets $\Lambda^{2}=\omega_{0}^{2}-\Omega^{2}$, the effective potential becomes

$$
V_{\mathrm{eff}}(x, y)=m\left(\omega_{0}^{2}-\Omega^{2}\right) x^{2}+\frac{1}{4} u x^{4},
$$

leaving no $y$ dependence in it. This means that the system will reach a state under extreme elongation along the $y$ direction. This is the case of most interest in the current context (effective 1D case). Nevertheless, for practical reason, we will assume that the system is confined in a large rectangular box of side length $L$. When $u=0$ (no quartic trap), as mentioned before, the system will become unstable for $\Omega \geq \omega_{0}$ unless the $s$-wave interaction is attractive $\left(g_{2 \mathrm{D}}<0\right)$. However when $u \neq 0$, the system remains stabilized for $\Omega \geq \omega_{0}$ and $g_{2 \mathrm{D}}>0$.

To solve the wavefunction of the system, we shall use an approach similar to that used in Refs. 22, 23. Firstly, we apply the gauge transformation, $\Psi_{0}=\Psi e^{-i m \Omega x y / \hbar}$, and consequently the energy functional (4) becomes

$$
\begin{aligned}
E & =\int d x d y \Psi^{*} \\
& \times\left[-\frac{\hbar^{2} \nabla^{2}}{2 m}+V_{\mathrm{eff}}^{\prime}(x, y)+\frac{g_{2 \mathrm{D}}}{2}|\Psi|^{2}+2 i \hbar \Omega x \frac{\partial}{\partial y}\right] \Psi
\end{aligned}
$$

with $V_{\text {eff }}^{\prime}(x, y)=m\left(\omega_{0}^{2}+\Omega^{2}\right) x^{2}+(u / 4) x^{4}$. Secondly the $s$-wave interaction $g_{2 \mathrm{D}}$ is assumed to be weak such that wavefunctions corresponding to no interaction case $\left(g_{2 \mathrm{D}}=0\right)$ can be used as the basis functions. Thirdly, the effect of the $s$-wave interaction will be reinstalled and treated by the perturbation method. Thus it is proposed that the wave function $\Psi$ is a linear combination of the lowest level eigenfunction(s), $\phi_{k}$, associated with energy functional (6) with $g_{2 \mathrm{D}}=0$. That is,

$$
\Psi=\sqrt{N} \sum_{k} C_{k} \phi_{k}
$$

where $N$ is the total number of particles and it requires that $\sum_{k}\left|C_{k}\right|^{2}=1$ for completeness. Here $k \equiv 2 \pi \ell / L$ with $\ell$ an integer. Since $L$ is large, $k$ can be treated in the continuous limit. When the quartic trap vanishes $(u=$ $0), \phi_{k}$ corresponds to LLL. Furthermore, eigenfunctions $\phi_{k}$ are assumed to take the separate form

$$
\phi_{k}=A e^{i k y} \chi_{k}(x),
$$

where $A=1 / \sqrt{L}$ is the normalization constant. Substituting Eqs. (7) and (8) into Eq. (6) with $g=0$, one obtains the one-dimensional (dimensionless) differential equation for $\chi_{k}(x)$,

$$
\begin{array}{r}
{\left[-\frac{d^{2}}{d x^{2}}+\left(2+\frac{2 \Omega^{2}}{\omega_{0}^{2}}\right) x^{2}-\frac{4 \Omega}{\omega_{0}} k x+\lambda x^{4}+k^{2}\right] \chi_{k}} \\
=\varepsilon_{k} \chi_{k},(9
\end{array}
$$

where $\varepsilon_{k}$ is the eigenenergy. Here $\lambda \equiv u \hbar / 2 m^{2} \omega_{0}^{3}$ and all the lengths and energies are scaled by the unit length $a_{0} \equiv \sqrt{\hbar / m \omega_{0}}$ and unit energy $E_{0} \equiv \hbar \omega_{0} / 2$. When $\lambda=0$, one has analytic solutions for Eq. (9) [23]. But for $\lambda \neq 0$, no analytic solution is available and numerical computation is needed. Here we shall apply the finite difference method to find solutions of the normalized function $\chi_{k}$. 

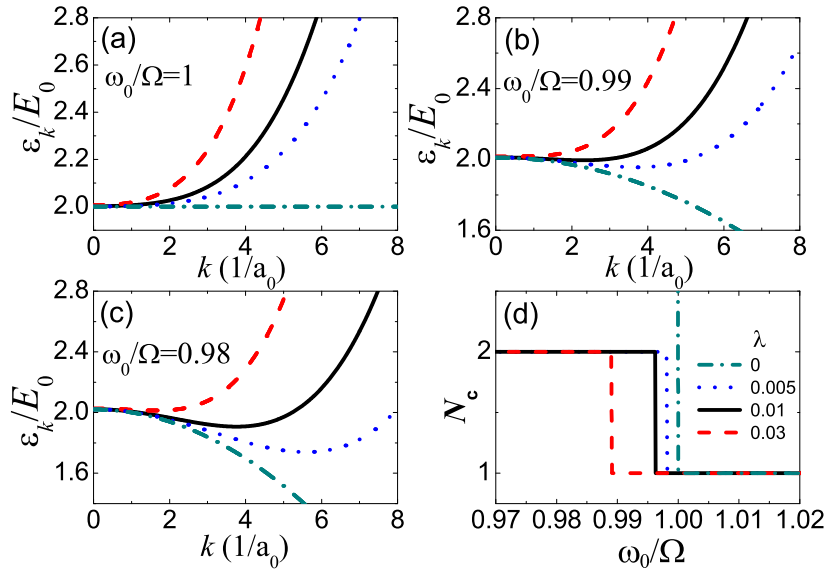

FIG. 1. (Color online) In frame (a)-(c), lowest eigenenergy of Eq. (9), $\varepsilon_{k}$, is plotted as the function of $k$ for three cases: $\omega_{0} / \Omega=1.0,0.99$ and 0.98 respectively. Frame (d) plots $N_{c}$ (see text) as the function of $\omega_{0} / \Omega$. In each frame, the four lines correspond to $\lambda=0.0,0.005,0.01$, and 0.03 respectively. For the units, $E_{0}=\hbar \omega_{0} / 2$ and $a_{0}=\sqrt{\hbar / m \omega_{0}}$.

With Eqs. (7)-(9), the full GP energy functional (6) becomes

$$
\begin{aligned}
\frac{E}{N} & =\sum_{k}^{N_{c}}\left|C_{k}\right|^{2} \varepsilon_{k}+\frac{n g}{2} \sum_{k_{1}, k_{2}, k_{3}, k_{4}}^{N_{c}} C_{k_{1}}^{*} C_{k_{2}}^{*} C_{k_{3}} C_{k_{4}} \\
& \times \int d x \chi_{k_{1}} \chi_{k_{2}} \chi_{k_{3}} \chi_{k_{4}} \delta_{k_{3}+k_{4}, k_{1}+k_{2}},
\end{aligned}
$$

where $n \equiv N /\left(L / a_{0}\right), g \equiv 4 \sqrt{2 \pi} a_{s} / a_{z}$ represents the new scale of $g_{2 \mathrm{D}}$, and $N_{c}$ is the number of terms used in the linear combination. $N_{c}$ is determined upon the condition that energy functional is minimized. The first part of the GP functional (10) involves the kinetic, harmonic, anharmonic (quartic), and rotational energies. While the second part corresponds to the interaction energy obtained by the perturbation manner. It is worth noting in Eq. (9) that the function $\chi_{k}(x)=\chi_{-k}(-x)$ and the energy $\varepsilon_{k}=\varepsilon_{-k}$. Due to the symmetry of $\varepsilon_{k}=\varepsilon_{-k}$, it is useful to write Eq. (7) as

$$
\Psi=\sqrt{N}\left[C_{0} \phi_{0}+\sum_{i=1}^{j}\left(C_{k_{i}} \phi_{k_{i}}+C_{-k_{i}} \phi_{-k_{i}}\right)\right] .
$$

With Eq. (11), it's evident that there are two cases for $N_{c}$. One is $N_{c}=2 j+1$ if $C_{0} \neq 0$ and another is $N_{c}=2 j$ if $C_{0}=0$.

\section{NONINTERACTING SYSTEM}

In this section, we consider the ground-state properties of a noninteracting system $(g=0)$. It will be shown for this noninteracting system that when $\lambda \neq 0$, the properties of the system are quite different from those of the $\lambda=0$ case. It is useful to first review the results for the $\lambda=0$ case 23]. When $\lambda=0$, one has the analytic solution for energy $\varepsilon_{k}$ in Eq. (9)

$$
\varepsilon_{k} \rightarrow \varepsilon_{k}^{0}=\sqrt{2\left(1+\frac{\Omega^{2}}{\omega_{0}^{2}}\right)}+k^{2}\left(\frac{1-\frac{\Omega^{2}}{\omega_{0}^{2}}}{1+\frac{\Omega^{2}}{\omega_{0}^{2}}}\right) .
$$

The results of Eq. (12) can be divided into three folds. (i) When $\Omega<\omega_{0}$, minimum $\varepsilon_{k}^{0}$ occurs at $k=0$. Consequently the ground-state wavefunction of the system is just $\Psi=\sqrt{N} C_{0} \phi_{0}\left(N_{c}=1\right)$. (ii) When $\Omega=\omega_{0}, \varepsilon_{k}^{0}$ is the same for all $k$ 's. In this highly degenerate case, the ground-state wavefunction should include all eigenstates $\left(N_{c}=\infty\right)$. (iii) When $\Omega>\omega_{0}$, minimum $\varepsilon_{k}^{0}$ occurs at $k \rightarrow \infty$. It indicates that the system is unstable. An important consequence of the $g=\lambda=0$ case is that vortex (lattice) will not form regardless of the ratio of $\omega_{0} / \Omega$.

When $\lambda \neq 0$, in contrast, behaviors of the system can be very different. We first consider the case when $\lambda$ is small such that wavefunction of the $\lambda=0$ case can be used to obtain the $\lambda$ correction perturbatively. The leading correction of the energy is found to be $\left(\varepsilon_{k} \simeq \varepsilon_{k}^{0}+\Delta \varepsilon_{k}^{0}\right)$

$$
\Delta \varepsilon_{k}^{0}=\lambda\left(A_{0}+A_{2} k^{2}+A_{4} k^{4}\right),
$$

where $A_{0}=3 /(8 R), A_{2}=3 \Omega^{2} /\left(\sqrt{2} \omega_{0}^{2} R^{5 / 2}\right)$, and $A_{4}=$ $\Omega^{4} /\left(\omega_{0}^{4} R^{4}\right)$ with $R \equiv 1+\Omega^{2} / \omega_{0}^{2}$. Note that all three $A_{i}$ are positive, so the correction $\Delta \varepsilon_{k}^{0}>0$. Moreover, it is found that the behaviors of the wavefunction $\Psi$ can be divided into two branches. When $\Omega \leq(1+3 \lambda / 8) \omega_{0}$, minimum $\varepsilon_{k}$ occurs at $k=0$. Consequently $\Psi=\sqrt{N} C_{0} \phi_{0}$ $\left(N_{c}=1\right)$. In contrast when $\Omega>(1+3 \lambda / 8) \omega_{0}, \Psi=$ $\sqrt{N}\left(C_{k_{1}} \phi_{k_{1}}+C_{-k_{1}} \phi_{-k_{1}}\right)\left(N_{c}=2\right)$, where $k_{1}$ corresponds to where minimum $\varepsilon_{k}$ is. Comparing these small but finite $\lambda$ results to those of $\lambda=0$ discussed before, one sees clearly that the general behaviors are quite different between the $\lambda=0$ and $\lambda \neq 0$ cases, especially in the regime $\Omega \gtrsim \omega_{0}$.

When $\lambda$ is not too small such that perturbation approach is no longer valid, one needs to solve Eq. (9) numerically for $\varepsilon_{k}$. In Fig. 1(a)-(c) with $\omega_{0} / \Omega=1.0,0.99$, and 0.98 respectively, we numerically solve and plot $\varepsilon_{k}$ as the function of $k$. Three finite $\lambda$ cases $(\lambda=0.005$, 0.01 , and 0.03$)$ are considered. The $\lambda=0$ case is included for comparison. Based on the experimental data given in Ref. [14] that $w_{x} \sim 2 \pi \times 64.8 \mathrm{~Hz}$ corresponds to $\lambda \simeq 0.001$, it is estimated that $w_{x} \sim 2 \pi \times 38.5,2 \pi \times 30$, and $2 \pi \times 21 \mathrm{~Hz}$ will correspond to $\lambda=0.005,0.01$, and 0.03 cases respectively. As shown in Fig. 1(a) for $\omega_{0} / \Omega=1.0, k_{1}$ (corresponding to minimum $\varepsilon_{k}$ ) is zero for all three finite $\lambda$ cases. This is in big contrast to the $\lambda=0$ case where $\varepsilon_{k}^{0}$ is $k$ independent [see Eq. (12)]. In the case of $\omega_{0} / \Omega=0.99, k_{1}$ is finite for $\lambda=0.01$ and 0.005 cases but is zero for the $\lambda=0.03$ case [see Fig. 1(b)]. While the corresponding $\varepsilon_{k}^{0}$ becomes lesser when $k$ is larger and no minimum-energy state is found. Finally for the case 
(a) $\omega_{0} / \Omega=1.0$

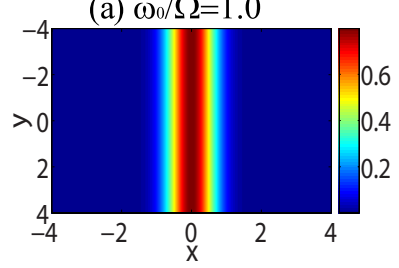

(c) $\omega_{0} / \Omega=0.993$

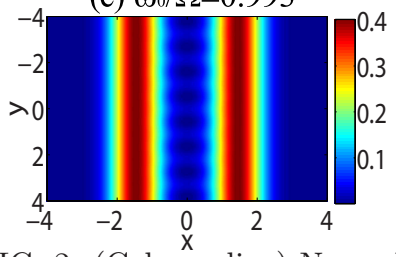

FIG. 2. (Color online) Normalized atom number density distribution, $|\Psi|^{2} / n$, is plotted in the $x y$ plane for (a) $\omega_{0} / \Omega=$ 1.0 , (b) 0.995 , (c) 0.993 , and (d) 0.99 . Here $\lambda$ is 0.005 for all frames. As shown, single vortex line exhibits in frame (b) and (c) only. $x$ and $y$ axes are in units of $a_{0}=\sqrt{\hbar / m \omega_{0}}$.

of $\omega_{0} / \Omega=0.98$ [see Fig. 1(c)], $k_{1}$ is finite for three $\lambda \neq 0$ cases. Similar to the $\omega_{0} / \Omega=0.99$ case, $\varepsilon_{k}^{0}$ will become lesser when $k$ is larger and no minimum-energy state is found. Thus for the larger $\lambda(=0.03)$ case, vortex forms only when the angular velocity $(\Omega)$ is larger. Moreover with the same $\lambda$, the value of $k_{1}$ (corresponding to minimum $\varepsilon_{k}$ ) is larger when $\Omega$ is larger. This means that the number of vortices per unit length (in the elongated direction) will be larger when $\Omega$ is larger. In Fig. 11(d), $N_{c}$ is plotted as the function of $\omega_{0} / \Omega$ for different $\lambda$ cases.

In practice, it is useful to compare the energy scale of the (dimensionless) quartic coupling $\lambda$ to the harmonic trap frequency $\omega_{x}$ or $\omega_{y}$. When $\Omega \sim \omega_{0}, \omega_{x}^{2} / \omega_{0}^{2}=2-$ $\left(\omega_{0} / \Omega\right)^{2} \sim 1$. Since the results discussed above are for $\lambda \lesssim 0.03$, the effect of the quartic trap is seen to be quite drastic.

In Fig. 2] normalized atom number density distributions, $|\Psi|^{2} / n$, are plotted in the $x y$ plane. Four cases are considered, namely $\omega_{0} / \Omega=1.0,0.995,0.993$, and 0.99 with $\lambda=0.005$ in all cases. In the case of $\omega_{0} / \Omega=1.0$ [Fig. $2(\mathrm{a})$ ], no vortex is formed. When the rotation velocity $\Omega$ is increased, single vortex line is seen to occur for both the $\omega_{0} / \Omega=0.995$ and 0.993 cases [Fig. 2(b) and (c)]. It is noted, however, that number of vortices per unit length is larger for the $\omega_{0} / \Omega=0.993$ case as compared to that of the $\omega_{0} / \Omega=0.995$ case. When $\Omega$ is further increased to above a critical value, $\omega / \Omega_{c} \lesssim 0.99$, vortex state disappears (melts) due to the large centrifugal force [see Fig $2(\mathrm{~d})]$. At this large $\Omega$ case, atoms are pushed to two sides along the elongated potential well and no atoms are left in the center.

The most important results obtained in this section for the noninteracting system $(g=0)$ are summarized as the following. (i) The quartic trap can make the system remain stable at higher rotation velocity $\left(\Omega>\omega_{0}\right)$. (ii) Single vortex line can exhibit at some $\Omega$ regimes. (iii) The single-line vortex lattice will vanish eventually at some
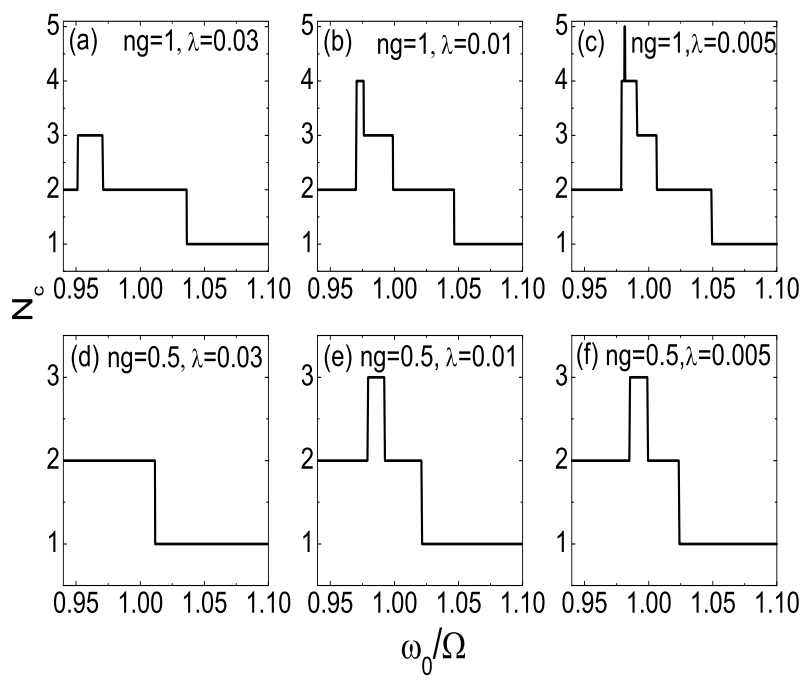

FIG. 3. $N_{c}$ is plotted as the function of $\omega_{0} / \Omega$ for six cases: (a) $(n g, \lambda)=(1,0.03)$, (b) $(1,0.01),(\mathrm{c})(1,0.005)$, (d) $(0.5,0.03)$, (e) $(0.5,0.01)$ and (f) $(0.5,0.005)$. Transition states occur in all frames except $(\mathrm{d})$.

higher $\Omega$ to which atoms are push to two sides along the elongated potential well. In next section, the effect of interaction $(g \neq 0)$ will be discussed in details.

\section{EFFECT OF INTERACTION}

In this section, the effect of interaction $(g)$ is studied by the perturbation approach. Similar to previous section, we consider also the three finite $\lambda$ cases: $\lambda=0.03$, 0.01 , and 0.005 . When the energy associated with the quartic trap is smaller than the energy associated with the harmonic trap and when $\Omega \sim \omega_{0}$, the energy difference between lowest and first excited band of $\varepsilon_{k}$ is about $2 \hbar \omega_{0}$. In our dimensionless scale, $2 \hbar \omega_{0}=4$. Thus the perturbative approach is valid as long as

$$
\frac{g}{2}|\Psi|^{2} \ll 4 \quad \text { or } \quad n g\left(\frac{|\Psi|^{2}}{n}\right) \ll 8 .
$$

The value of $|\Psi|^{2} / n$ is roughly 0.4 (see Figs. 2 and 5 ). Thus when $n g \ll 20$, the perturbation approach is valid. Hence the values of $n g=0.5$ and 1.0 considered here should be safely valid for the perturbative approach. Typically $n$ is more than 1 in a $2 \mathrm{D}$ system [22]. If $N=10^{4}$ and $L / a_{0}=100$, the value of $n$ is about 100 . For $n g=1$, it corresponds to $a_{s} / a_{z} \simeq 10^{-3}$. In Fig. 3. the number of $N_{c}$ [terms involved in the summation (10]) is determined and plotted as the function of $\omega_{0} / \Omega$ for six cases, namely $(n g, \lambda)=(1,0.03),(1,0.01),(1,0.005)$, $(0.5,0.03),(0.5,0.01)$, and $(0.5,0.005)$. It turns out that the results of $N_{c}$ are quite fascinating in terms of the change of $\Omega$ and the interplay between the strength of the interaction and the strength of the quartic potential. 


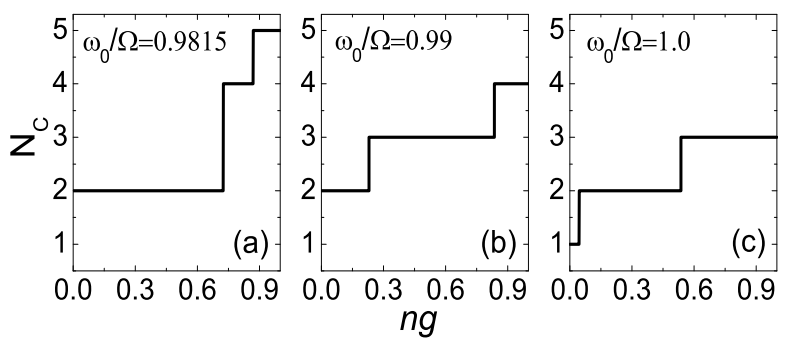

FIG. 4. $N_{c}$ is plotted as the function of $n g$ for three cases: (a) $\omega_{0} / \Omega=0.9815$, (b) 0.99 , and (c) $1.0 . \lambda=0.005$ in all three frames.

One common feature of all frames in Fig. 3 is that $N_{c}$ shifts from 1 (at lower $\Omega$ ) to 2 eventually (at higher $\Omega$ ). In between of the 1 to 2 period, transition states occur for a large $\Omega$ span. For example, $N_{c} \geq 3$ transition states can occur in all frames except in frame (d). Besides, $N_{c}$ increases in a trend as $\Omega$ increases for the transition states. The reason why $N_{c}$ all shift from 1 (at lower $\Omega$ ) to 2 eventually (at higher $\Omega$ ) can be understood as follows. One recalls the $N_{c}$ results for $g=0$ in Fig. 1(d), where all three finite $\lambda$ cases are observed to exhibit the $N_{c}=1 \rightarrow 2$ transition (at different critical $\Omega$ though).

When the interaction $g$ is turned on and still valid in the perturbative regime, $g$ will only play little role in the large $\Omega$ limit. It means that when $\Omega$ is large enough, transition states, which arise due to the effect of $g$, will disappear. As an extreme example shown in Fig. 3(d), because $g$ is relatively small $(n g=0.5)$ and the quartic coupling is relatively large $(\lambda=0.03)$, consequently no transition state occurs. When $\lambda$ is reduced [see Fig. 3(e) and (f)], or when $g$ is increased [see Fig. 3(a)], transition states will occur.

Moreover, it is found that the critical value of $\Omega$ to which $N_{c}$ changes from 1 to 2 at the lower $\Omega$ side is smaller when $\lambda$ is smaller (if $n g$ is fixed) or when $n g$ is larger (if $\lambda$ is fixed). It implies that the system will enter the vortex state earlier if $g$ is relatively larger or $\lambda$ is relatively smaller. Furthermore, with the same $\lambda$, transition states will sustain for a larger span of $\Omega$ if $g$ is larger and can go up to a higher value of $N_{c}$. The latter means that vortex lattice can have a higher row number. One sees in Fig. 3(c) that $N_{c}$ can go up to 5 , although for a small period of $\Omega$. Fig. 4 plots number $N_{c}$ as the function of $n g$ with $\lambda=0.005$ and $\omega_{0} / \Omega=0.9815$, 0.99 , and 1.0 respectively. Basically Figs. 3 and 4 show how the different regimes behave as the change of the interaction, quartic trap strength, and rotation velocity.

The normalized density profile in the $x y$ plane, $|\Psi|^{2} / n$, is shown in Fig. 5 for $(n g, \lambda)=(1,0.005)$ [corresponding to the case in Fig. 3(c)]. As seen in Fig. 31(c), the change of $N_{c}$ in the transition states is quite rich for this case. Six angular velocities are studied, namely $\omega_{0} / \Omega=1.02,1.0$, $0.984,0.9815,0.98$ and 0.9785 respectively for Fig. 5 (a)(f). With these values of $\Omega, N_{c}$ corresponds to $2,3,4$, (a) $\omega_{0} / \Omega=1.02, \mathrm{~N}_{\mathrm{c}}=2$

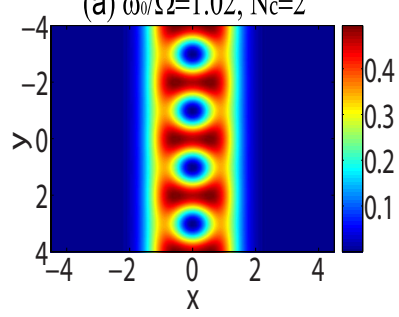

(c) $\omega_{0} / \Omega=0.984, \mathrm{~N}_{\mathrm{c}}=4$

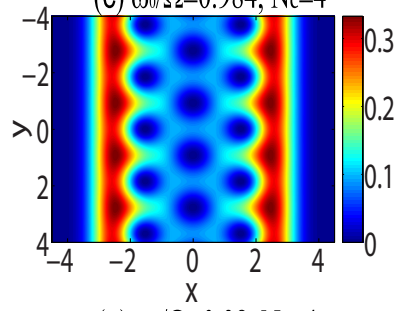

(e) $\omega_{0} / \Omega=0.98, \mathrm{~N}_{\mathrm{c}}=4$
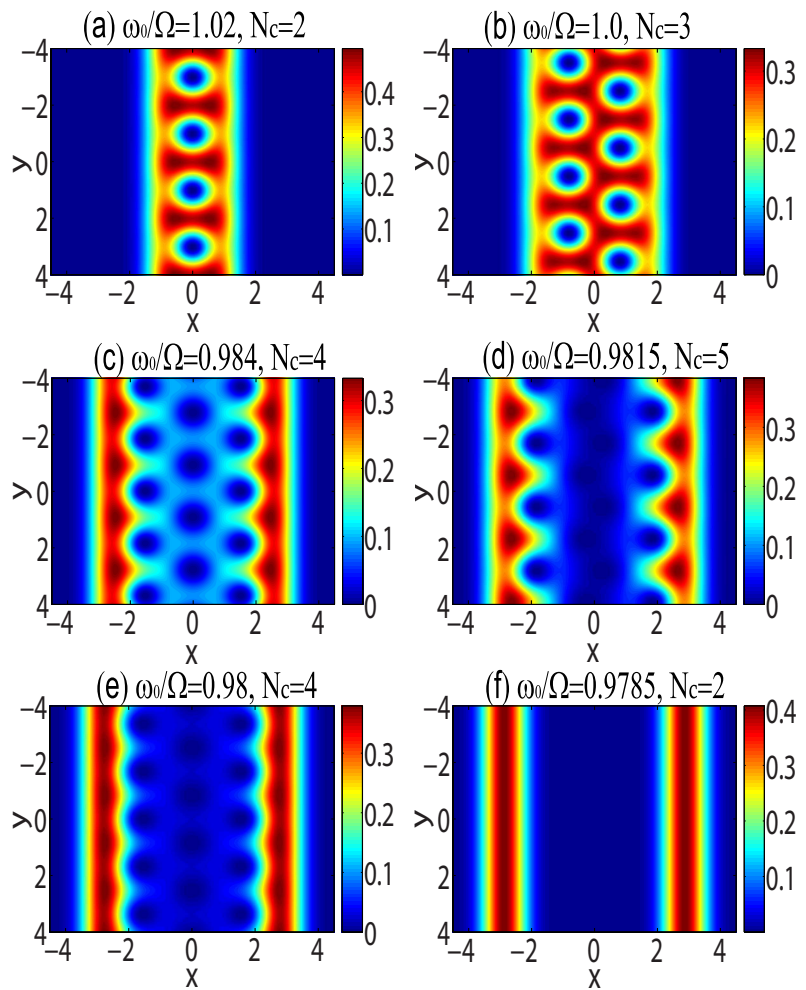

(d) $\omega_{0} / \Omega=0.9815, \mathrm{~N}_{\mathrm{c}}=5$

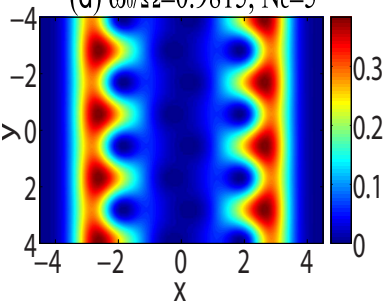

(f) $\omega_{0} / \Omega=0.9785, \mathrm{~N}_{\mathrm{c}}=2$

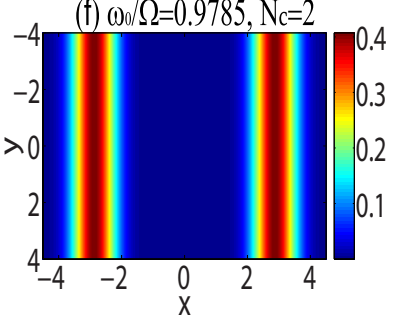

FIG. 5. (Color online) Normalized atom number density distribution, $|\Psi|^{2} / n$, is plotted in the $x y$ plane for (a) $\omega_{0} / \Omega=$ 1.02 , (b) 1.0 , (c) 0.984 , (d) 0.9815 , (e) 0.98 , and (f) 0.9785 . Here $n g=1$ and $\lambda=0.005$ for all frames [corresponding to the case in Fig. 3(c)]. Vortex lattices with the transitions of row number and lattice constant are observed as $\Omega$ changes. $x$ and $y$ axes are in units of $a_{0}=\sqrt{\hbar / m \omega_{0}}$.

5, 4, and 2 respectively. One sees in Fig. $5(\mathrm{a})-(\mathrm{d})$ that when $\Omega$ is increased from $\omega_{0} / \Omega=1.02$ to 0.9815 , atoms are pushed to the two sides and the number of vortices becomes more and more. The row number of vortex line also increases from 1 to 4 . While in Fig. 5 (e), the vortex row number is reduced to $3\left(N_{c}=4\right)$ again. In the case of Fig. 5 (f), although $N_{c}$ is reduced to 2, but vortex lattice vanishes (melts) due to the large centrifugal force. Similar vortex lattice melting transition (atoms are completely pushed to the two sides) at large $\Omega$ has already been seen in the previous section of no interaction.

\section{CONCLUSIONS}

This paper investigates the effect of a quartic potential on a fast rotating BEC system under the extreme elongation. In contrast to the harmonic trap alone case where system is unstable when the angular velocity $\Omega$ is larger than the radial trap oscillator frequency $\omega_{0}$, the quartic trap can lead the system to remain stable at higher rotation velocity $\left(\Omega>\omega_{0}\right)$. The interplay between the weak $s$-wave interaction and the quartic trap potential can result rich vortex lattice transition states as a function of 
$\Omega$. At large $\Omega$, atoms are eventually push to the two sides along the elongated potential well.

\section{ACKNOWLEDGMENTS}

This work is supported by the National Science Council, Taiwan under the Grant No. 96-2112-M-003-008. We also acknowledge the support from NCTS, Taiwan.
[1] M.R.Matthews, B.P.Anderson, P.C.Haljan, D.S.Hall, C.E.Wieman, and E.A. Cornell, Phys. Rev. Lett. 83, 2498 (1999).

[2] K. W. Madison, F. Chevy, W. Wohlleben, and J. Dalibard, Phys. Rev. Lett. 84, 806 (2000).

[3] J. R. Abo-Shaeer, C. Raman, J. M. Vogels, and W. Ketterle, Science 292, 476 (2001).

[4] C. Raman, J. R. Abo-Shaeer, J. M. Vogels, K. Xu, and W. Ketterle, Phys. Rev. Lett. 87, 210402 (2001).

[5] P. C. Haljan, I. Coddington, P. Engels, and E. A. Cornell, Phys. Rev. Lett. 87, 210403 (2001).

[6] P. Engels, I. Coddington, P. C. Haljan, and E. A. Cornell, Phys. Rev. Lett. 89, 100403 (2002).

[7] V. Schweikhard, I. Coddington, P. Engels, V.P. Mogendorff, and E.A. Cornell, Phys. Rev. Lett. 92, 040404 (2004).

[8] Tin-Lun Ho, Phys. Rev. Lett. 87, 060403 (2001).

[9] Gordon Baym and C. J. Pethick, Phys. Rev. A 69, 043619 (2004).

[10] N. R. Cooper and S. Komineas, Phys. Rev. A 70, 033604 (2004).

[11] Gentaro Watanabe, Gordon Baym, and C. J. Pethick, Phys. Rev. Lett. 93, 190401 (2004).

[12] A. Aftalion, X. Blanc, and J. Dalibard, Phys. Rev. A 71, 023611 (2005).
[13] Gentaro Watanabe, S. Andrew Gifford, Gordon Baym, and C. J. Pethick, Phys. Rev. A 74, 063621 (2006).

[14] V. Bretin, S. Stock, Y. Seurin, and J. Dalibard, Phys. Rev. Lett. 94, 050403 (2004).

[15] X. Blanc and N. Rougerie, Phys. Rev. A. 77, 053615 (2008).

[16] A. D. Jackson and G. M. Kavoulakis, Phys. Rev. A 70, 023601 (2004).

[17] A. D. Jackson, G. M. Kavoulakis, E. Lundh, Phys. Rev. A 69, 053619 (2004).

[18] A. L. Fetter, Phys. Rev. A 64, 063608 (2001).

[19] A. L. Fetter, B. Jackson, and S. Stringari, Phys. Rev. A 71, 013605 (2005)

[20] M. Ö. Oktel, Phys. Rev. A 69, 023618 (2004).

[21] A. L. Fetter, Phys. Rev. A 75, 013620 (2007).

[22] S. Sinha and G. V. Shlyapnikov, Phys. Rev. Lett. 94, 150401 (2005).

[23] P. Sánchez-Lotero and J. J. Palacios, Phys. Rev. A 72, 043613 (2005).

[24] S. I. Matveenko, D. Kovrizhin, S. Ouvry, and G. V. Shlyapnikov, Phys. Rev. A 80, 063621 (2009).

[25] D. S. Petrov, M. Holzmann, and G. V. Shlyapnikov, Phys. Rev. Lett. 84, 2551 (2000). 